\title{
THE MOST GENERAL TRANSFORMATIONS OF PLANE REGIONS WHICH TRANSFORM CIRCLES INTO CIRCLES
}

\section{BY CONSTANTIN CARATHÉODORY}

1. Introduction. If we consider two plane regions $R$ and $R^{\prime}$ which are mapped conformally the one upon the other, there corresponds to every circle $c$ contained in $R$ an analytic closed curve $c^{\prime}$ contained in $R^{\prime}$. If an arc $\alpha$ lying on the circle $c$ has a circular image the curve $c^{\prime}$ must be itself a circle.

Suppose that the interior of the circle $c$ belongs to the region $R$. Then both circular discs bounded by $c$ and $c^{\prime}$ respectively are represented conformally the one upon the other. It is a well known fact that in this case the transformation of these circles into one another is given by a transformation of $M$ bius by which, furthermore, every circle of $R$ is transformed into a circle of $R^{\prime}$.

If we drop now the condition that the one to one correspondence of our regions must be conformal, the assumption that one single circle of $R$ has a circular image is no longer sufficient to characterize the transformations of Möbius. On the other hand, if we make the stronger assumption that every closed circle contained in $R$ is transformed into a circle, we shall see in the course of this paper that a theorem analogous to the one stated above holds under surprisingly general conditions. To prove that the transformation which we consider is a transformation of Möbius it is no longer necessary to assume from the outset (as is the case for the analogous theorem concerning collinear transformations) that this transformation is continuous, or that it is measurable in the sense of Lebesgue, or even that the point set $R^{\prime}$ is itself a region.

The condition that circles lying in a region $R$ have circular images characterizes the group of Möbius transformations among all the one to one arbitrary correspondences between the points of $R$ and the points of a quite arbitrary point set of the plane.

2. Statement of a Preliminary Theorem. We consider a circular open disc which we shall call $C$ and suppose that there is a one 
to one correspondence between the points $P$ of this disc and the points $P^{\prime}$ of a bounded point set $C^{\prime}$. We do not suppose this correspondence to be continuous or even to be measurable in the sense of Lebesgue. But we assume that every closed circular line $c$ which is contained in the domain $C$ is transformed by the above correspondence into a line $c^{\prime}$ of the same kind whose points lie on $C^{\prime}$. We shall prove that every transformation which possesses these properties is a Möbius transformation and therefore analytic.

It is very important to make the following rather obvious remark: if two circles $c_{1}^{\prime}$ and $c_{2}^{\prime}$ contained in $C^{\prime}$ are the images of two circles of $C$, say of $c_{1}$ and $c_{2}$, then the number of points common to the pair of circles $c_{1}^{\prime}$ and $c_{2}^{\prime}$ is the same as the number of points common to $c_{1}$ and $c_{2}$. In particular, if $c_{1}$ and $c_{2}$ are tangent to one another the same is the case for $c_{1}^{\prime}$ and $c_{2}^{\prime}$ and conversely.

3. Reduction of the Transformation to a Normal Type. In using transformations of Mobius we plot the domain $C$ and a circle $D^{\prime}$ which contains in its interior the bounded point set $C^{\prime}$ on the unit circles of two planes with coordinates $x, y$ and $x^{\prime}, y^{\prime}$ respectively. In doing this we can always assume that the origins of both planes correspond to points of $C$ and of $C^{\prime}$ which are images of one another by the given transformation.

Finally we make two inversions respectively on the unit circles of both these planes.

4. Preservation of Parallelism. To the original transformation of the domain $C$ into the point set $C^{\prime}$ corresponds now a one to one transformation of the exterior $E$ of the unit circle in the plane with coordinates $x, y$ into some point set $E^{\prime}$ lying outside of the unit circle of the second plane. To every closed circular line lying in $E$ corresponds a closed circle of $E^{\prime}$. Finally every straight line contained entirely in $E$ is transformed into a straight line lying in $E^{\prime}$.

Likewise every pair of parallel straight lines of $E$ is transformed into a pair of parallel straight lines of $E^{\prime}$.

5. Preservation of Orthogonality. Take now two such pairs of parallel straight lines lying in $E$ and cutting at right angles. Suppose furthermore that there is a circle contained entirely in $E$ and circumscribed to the rectangle formed by these lines. 
The whole figure is transformed into a circle of $E^{\prime}$ in which a parallelogram is inscribed; this parallelogram must therefore be a rectangle.

We infer herefrom without difficulty that two straight lines of $E$ orthogonal to one another are always transformed into lines of $E^{\prime}$ having the same property.

6. The Image of $C$ is a Domain. We take two circles of $E$ which are concentric to the unit circle and consider two or more rectangles inscribed in the one of these circles and having two opposite sides tangent to the other. As the corresponding figure lying on $E^{\prime}$ shows exactly the same disposition, the circles contained in these figures and which are images of the two circles considered above must also be concentric to one another.

Furthermore let $c$ be an arbitrary circle of $E$ concentric to the unit circle. To every point $P^{\prime}$ lying on the image $c^{\prime}$ of $c$ corresponds a point $P$ of $c$. To the tangent to the circle $c$ passing through the point $P$ corresponds the tangent to $c^{\prime}$ passing through $P^{\prime}$. Consequently every point of the plane with coordinates $x^{\prime}, y^{\prime}$ exterior to $c^{\prime}$ must belong to the point set $E^{\prime}$. We conclude from this that $E^{\prime}$ is an open set consisting of all the points which are exterior to a certain circle. The point set $C^{\prime}$ which we have considered above must therefore be a circular disc and we could have taken the circle $D^{\prime}$ coinciding with the boundary of $C^{\prime}$.

If we do this the point set $E^{\prime}$ coincides with the exterior of the unit circle of our plane with coordinates $x^{\prime}, y^{\prime}$, and the circles of $E$ concentric to the unit circle are transformed into circles of $E^{\prime}$ having the same property.

7. Preservation of the Centers of Circles. Take now a circle $\gamma$ lying in $E$ whose center is at a distance from the origin greater than $2^{1 / 2}$. Under these conditions there are at least two diameters of $\boldsymbol{\gamma}$ orthogonal to one another which belong to straight lines lying in $E$. There are also at least two tangents to the same circle cutting at right angles and parallel to the diameters which we have been considering and entirely contained in $E$.

This figure transforms into a figure contained in $E^{\prime}$ from whose inspection we conclude that if we call $\gamma^{\prime}$ the image of $\gamma$, the center of $\gamma^{\prime}$ is the image of the center of $\gamma$. 
8. Preservation of Angles at the Origin. We take now the positive number

$$
r>2^{1 / 2},
$$

and consider the circle

$$
x^{2}+y^{2}=r^{2}
$$

and the circle

$$
x^{\prime 2}+y^{\prime 2}=r^{\prime 2}
$$

into which the circle (2) is transformed. We will show that every regular polygon inscribed in (2) and whose sides have a length less than $(r-1)$ is transformed into a regular polygon inscribed in (3) and having the same number of vertices.

Two adjoining sides of the former polygon must in fact, by the result of the last paragraph, be transformed into contiguous segments of equal length inscribed in (3). The second polygon is therefore either a regular polygon similar to the first or it is starshaped. But in this latter case at least two sides of it would cross at a point interior to the circle (3) and such a point must necessarily be the image of a point exterior to the circle (2). But this is readily shown to be impossible by using a reasoning like that of $\S 6$. Thus it is proved that the two polygons are similar to one another.

We apply this to all such polygons inscribed in (2) which have a common vertex at the point $x=r, y=0$.

Any point of the circle (2) which satisfies the condition

$$
x+i y=r \cdot e^{2 \pi i p / q}
$$

where $p$ and $q$ are arbitrary integers and $q \neq 0$, can be considered as a vertex of one at least of these polygons. And we can always choose the coordinates $x^{\prime}$ and $y^{\prime}$ in such a way that the images of all the points (4) must be calculated by the formulas

$$
\frac{x^{\prime}}{r^{\prime}}=\frac{x}{r}, \quad \frac{y^{\prime}}{r^{\prime}}=\frac{y}{r} .
$$

9. Families of Circles which Are Transformed by a Similarity. Take now all the tangents to the circle (2) whose points of contact coincide with the point set (4) and call $A$ the set of points of intersection of each two of these tangents. Each point of the 
set $A$ will then be transformed into a certain point contained in the exterior of the circle (3) whose coordinates must be calculated by (5).

Call $A^{*}$ the family of circles, each of which is contained in $E$ and passes through three points at least of the set $A$. The image of every one of these circles is necessarily connected with the circle itself by the transformation (5).

We remark finally that the point set $A$ is everywhere dense in the exterior of the circle (2) and that therefore every circle lying in $E$ and possessing points outside of (2) can be indefinitely approximated by circles belonging to the family $A^{*}$.

10. Proof of the Continuity of the Transformation. Our next step is to show that our transformation is necessarily continuous along the circle (2). If this were not the case there would exist on this circle at least one point $P_{0}$ whose image on the circle (3) we obtain by a rotation around the origin through an angle $\theta$ lying between zero and $2 \pi$. We could then construct a point set $B$ analogous to our former point set $A$ and a set of circles $B^{*}$ analogous to $A^{*}$ and possessing the following property: To obtain the image of a circle belonging to $B^{*}$ we must combine the amplification of $\S 9$ according to the cases either with a rotation around the origin through the angle $\theta$ or with a reflection on some diameter of the circle (3).

In both these cases it is always possible to find two circles, say $c_{1}$ and $c_{2}$, both lying in the region

$$
x^{2}+y^{2}>r^{2},
$$

cutting one another, and such that if we transform $c_{1}$ by the amplification of $\S 9$ and $c_{2}$ by one of the transformations which have just been described, we obtain as result of these transformations circles which have no point in common.

We now approximate $c_{1}$ by a circle $c_{1}^{*}$ belonging to $A^{*}$ and $c_{2}$ by a circle $c_{2}^{*}$ belonging to $B^{*}$. If these approximations are close enough the circles $c_{1}^{*}$ and $c_{2}^{*}$ will cut one another and will be transformed by the transformation we study into circles having no common point. But this is in contradiction with the general principle laid down at the end of $\$ 2$.

We must therefore assume that all the points of the circle (2) are transformed into the points of (3) by the transformation 
(5) and the same is then the case for all the points of the region (6).

11. The Theorem for the Circle. Take now a point $P$ of $E$ lying between the unit circle and the circle (2). Consider two circles of $E$ passing through $P$ and possessing points inside of the region (6). By hypothesis these circles are transformed into circles and besides they contain arcs whose images we know.

The result is that the formulas (5) must hold for every point of the region $E$. As this region must by assumption be trans. formed into $E^{\prime}$ we find that we must have

$$
r^{\prime}=r .
$$

In short we have proved the following theorem:

THEOREM 1. Every arbitrary one to one correspondence between the points of a circular disc $C$ and a bounded point set $C^{\prime}$ by which circles lying completely in $C$ are transformed into circles lying in $C^{\prime}$ must always be either a direct or an inverse transformation of Möbius.

12. The General Theorem. We now consider a general region $R$ and suppose that there exists a one to one correspondence between the points of $R$ and some point set $R^{\prime}$. We assume furthermore that by this correspondence circles of $R$ are transformed into circles lying on $R^{\prime}$. We call $c$ and $c^{\prime}$ two such corresponding circles and suppose that every point interior to $c$ belongs to $R$. Through every pair of points $P$ and $Q$ lying in the interior of $c$ there passes at least one circle $c_{1}$ having no points in common with $c$. To this circle corresponds a circle $c_{1}^{\prime}$ which has no point in common with $c^{\prime}$. Therefore both points $P^{\prime}$ and $Q^{\prime}$ which correspond to $P$ and $Q$ must lie on the same side of $c^{\prime}$. It follows that the interior of $c$ must correspond to a point set which lies on one side of $c^{\prime}$. This point set is either itself bounded, or it can be transformed by an inversion on the circle $c^{\prime}$ into a bounded set. In both cases we can apply the previous theorem. As the circle $c$ can be taken at random we have finally the following theorem.

THEOREM 2. Suppose that to every point $P$ of a region $R$ corresponds a point $P^{\prime}$ of some point set $R^{\prime}$ and that to two different points $P$ and $Q$ of $R$ correspond two points $P^{\prime}$ and $Q^{\prime}$ of $R^{\prime}$ differ- 
ent from one another. Suppose that to every circle c contained with its interior in the region $R$ corresponds a point set of $R^{\prime}$ which consists of all the points of a closed circle $c^{\prime}$. Then the point set $R^{\prime}$ is itself a region and the transformation of $R$ into $R^{\prime}$ is analytic and either a direct or an inverse transformation of Möbius.

It is not difficult to generalize this result by restricting the class of circles $c$ belonging to $R$ which are supposed to be transformed into circles. Take for instance a continuous positive function $\phi(P)$ defined everywhere in the region $R$. Then the theorem holds if we suppose that every circle of center $P$ and whose radius is less than $\phi(P)$ is transformed into a circle lying on $R^{\prime}$.

The following generalization of Theorem 2 is nearly self evident if we note that three circles in space cutting one another at six different points must lie on the same sphere.

THEOREm 3. Supposing that a plane region $R$ is transformed by $a$ one to one correspondence into an arbitrary point set $R^{\prime}$ lying in $n$-space $(n \geqq 3)$ under the same assumptions as before, then $R^{\prime}$ must be a two dimensional sphere or a plane and the transformation is a transformation of Möbius.

Other generalizations can also be imagined which, however, are outside the scope of this note.

UNIVERSITY OF MUNICH

\section{A NOTE ON DETERMINANTS}

BY M. H. INGRAHAM

The following theorem, with its corollary, when applicable affords great numerical simplifications. Although the writer has tried and failed to discover it in the literature, it is submitted with some hesitation lest it may not be new. The corollary was discovered and proved by the author. Upon seeing it Professor Max Zorn suggested the more general form of the theorem, which was then proved by the author.

Theorem. If $A$ is a square matrix $\left(A_{i j}\right),(i, j=1, \cdots, n)$, where the $A_{i j}$ are $m \times m$ matrices which are commutative in pairs, and if $B$ is the $m \times m$ matrix which is arrived at by taking the 